doi:10.1017/\$1041610219002242

\section{Identifying and treating hearing loss in mild cognitive impairment: a feasibility study for an interventional trial with hearing aids}

Age-related hearing loss is an independent risk factor for dementia, with even mild hearing loss nearly doubling risk (Livingston et al., 2017). Observational studies find that wearing hearing aids may protect those with hearing loss from excess cognitive decline (Maharani et al., 2018). This suggests that treating hearing loss may deliver substantial reductions in dementia risk. People with mild cognitive impairment (MCI) who are at increased risk of dementia are typically diagnosed in their seventies (Sachdev et al., 2015), when age-related hearing loss is also common (Scholes and Mindell, 2015). A trial of hearing aids in those with MCI may clarify whether hearing aids can delay or prevent dementia. As more than $40 \%$ of new hearing aid users do not use them consistently or even at all, such a trial may be impractical because of low recruitment for hearing aids and poor retention (Aazh et al., 2015).

We aimed to find out if a trial in those with MCI and untreated hearing loss would be able to recruit and retain participants. We also looked at whether standard UK NHS pathways for hearing loss would be sufficient to ensure that potential participants have hearing aids prescribed within 3 months and that these are maintained long term.

We recruited 17 people aged $\geq 60$ years with ICD-10 diagnosis of MCI, from an NHS memory clinic in London, over 3 months. Twelve participants $(12 / 17=71 \%$ [95\% confidence interval $(\mathrm{CI})$ 49-92\%]) had hearing loss after a home-based assessment with a positive HearCheck screening result and confirmed in all cases as at least mild hearing loss by an audiologist though portable audiometry (pure tone average $(0.5-4 \mathrm{kHz})>25 \mathrm{~dB}$ HL). We sent the audiometric results to the participant's general practitioner (GP), with advice to refer to NHS audiological services (which have a 12-week target for fitting hearing aids) if appropriate. At 12 weeks follow-up, three participants had been referred by their GP for NHS audiological assessment (25\% [95\% CI: $1-49 \%])$. Of those, two received hearing aids $(2 / 12 ; 17 \%$ [95\% $1-38 \%])$. At 2 years follow-up, one out of eight contactable participants was still using their hearing aids $(12 \%$ [95\% CI: $1-35 \%])$.
Our study found that a high proportion of MCI participants attending an NHS memory clinic had untreated hearing loss. However, we also found that relying on the standard NHS audiological pathway, even after providing primary care with an audiological assessment consistent with hearing loss, resulted in low treatment uptake and low long-term adherence in people with MCI. This suggests that a trial of hearing aids in MCI could recruit participants, but that the usual audiological pathways would not be sufficient to find out whether they reduced cognitive loss. This has informed the Treating Auditory Impairment and Cognition pilot trial's intervention to include a home-based hearing assessment and hearing aid fitting, with a study audiologist and a research assistant providing motivational and practical advice and support over 3 months (TACT; https://www.ucl.ac.uk/psychiatry/tact-trial).

\section{Conflict of interest}

None.

\section{Description of authors' roles}

D. Proctor, S. Costafreda and D. Karia designed the study, carried out the study, interpreted data and drafted the paper. D.E. Bamiou, G. Lewis, G. Livingston, R. Omar and A. Schilder contributed to the design of the study, attended study meetings and contributed to revising the paper for intellectual content.

\section{Acknowledgements}

This work was supported by Alzheimer's Research UK Prevention and Risk Reduction Fund (ARUKPRRF2017-001), Camden and Islington NHS Foundation Trust and NOCLOR research support service (North Central London Research Consortium).

\section{Ethical approval}

The study was approved by an NHS Research Ethics Committee (REC reference: 16/WM/0518). All potential participants gave written informed consent. Participants were also contacted again two years later to assess whether they were using hearing 
aids or were potentially eligibility for the TACT pilot trial (REC reference: 18/LO/1196), an interventional trial with similar eligibility criteria.

\section{References}

Aazh, H., Prasher, D., Nanchahal, K. and Moore, B. C. (2015). Hearing-aid use and its determinants in the UK National Health Service: a cross-sectional study at the Royal Surrey County Hospital. International fournal of Audiology, 54(3), 152-161.

Livingston, G. et al. (2017). Dementia prevention, intervention, and care. The Lancet, 390(10113), 2673-2734.

Maharani, A., Dawes, P., Nazroo, J., Tampubolon, G. and Pendleton, N. (2018). Longitudinal relationship between hearing aid use and cognitive function in older Americans. Fournal of the American Geriatrics Society, 66(6), 1130-1136.

Sachdev, P.S. et al. (2015). The prevalence of mild cognitive impairment in diverse geographical and ethnocultural regions: the COSMIC collaboration. PLoS ONE, 10(11), e0142388.
Scholes, S. and Mindell, J. (2015). Hearing. In: R. Craig, E. Fuller, and J. Mindell (Eds.), Health Survey for England 2014. London: The Health and Social Care Information Centre.

Danielle Proctor, ${ }^{1}$ Doris-Eva Bamiou, ${ }^{2,3}$

Daniel Karia, ${ }^{3}$ Glyn Lewis, ${ }^{1}$ Gill Livingston, ${ }^{1}$ (D) Rumana Z. Omar, ${ }^{4}$ Anne G. M. Schilder ${ }^{2,3}$ AND Sergi Costafreda Gonzalez ${ }^{1}$

${ }^{1}$ UCL Division of Psychiatry, London, United Kingdom

${ }^{2}$ National Institute for Health Research, University College London Hospitals Biomedical Research Centre, London, United Kingdom

${ }^{3}$ UCL Ear Institute, London, United Kingdom

${ }^{4}$ UCL Department of Statistical Science, London, United Kingdom

Correspondence should be addressed to: Sergi Costafreda Gonzalez, Division of Psychiatry, University College London, 149 Tottenham Court Road, London, W1T 7NF, United Kingdom. Phone + 44 (0)207 6799059. Email: S.Costafreda@ucl.ac.uk. 